\title{
RELAÇÃO ENTRE PROCESSO DE TRABALHO E SAÚDE DE CAMINHONEIROS
}

\author{
Relationship Between Work Process and Health of Truckers \\ Relación del proceso de trabajo y la salud de camioneros
}

Artigo Original

\section{RESUMO}

Objetivo: Analisar a relação entre processo de trabalho e saúde de caminhoneiros do estado do Ceará. Métodos: Tratou-se de um estudo epidemiológico transversal desenvolvido com 165 caminhoneiros presentes no XX Festival dos Caminhoneiros do município de Tabuleiro do Norte-CE. Para a coleta de dados, aplicou-se um questionário com perguntas fechadas, abordando os temas relacionados ao processo de trabalho, saúde e estilo de vida. Os dados foram organizados em tabelas e analisados com o auxílio do programa EPI-INFO. Resultados: Constatou-se que $100 \%(n=165)$ dos profissionais eram do sexo masculino, 39,3\% $(n=65)$ encontravam-se na faixa etária de 30 a 39 anos, $40 \%(n=66)$ possuíam apenas o $1^{\circ}$ grau incompleto, 35,8\% $(\mathrm{n}=59)$ trabalhavam de 12 a 16 horas por dia. Os problemas de saúde mais comuns foram a insônia, presente em 78,1\% $(\mathrm{n}=129)$ dos caminhoneiros, apresentando relação significativa com o consumo de substâncias psicoativas $(\mathrm{p}=0,002)$; e dores lombares, presentes em 52,7\% $(n=87)$ deles. Conclusão: Existe uma grande exposição a fatores de risco imposta por essa profissão, podendo afetar de maneira significativa a execução do trabalho e a qualidade da saúde dos caminhoneiros. Portanto, tornam-se necessárias mudanças no estilo de vida deles e que as autoridades dirijam maior atenção para esses profissionais.

Descritores: Saúde do trabalhador; Estilo de Vida; Saúde do Viajante.

\section{ABSTRACT}

Objective: To analyze the relationship between the work process and the health of truckers in the state of Ceará. Methods: Epidemiological cross-sectional study conducted with 165 truck drivers attending the XX Truckers Festival of the municipality of Tabuleiro do NorteCE. Data were collected using a questionnaire with close-ended questions about issues related to the work process, health and lifestyle. Data were organized in tables and analyzed with the aid of EPI- INFO software. Results: It was verified that $100 \%(n=165)$ of the professionals were males, $39.3 \%(n=65)$ were aged $30-39$ years, $40 \%(n=66)$ had incomplete primary education, $35.8 \%(n=59)$ worked from 12 to 16 hours per day. The most common health problems were insomnia, which affected $78.1 \%(n=129)$ of the drivers and presented a significant association with the use of psychoactive substances ( $p=0.002)$; and lower back pain, which affected $52.7 \%(n=87)$ of the truckers. Conclusion: There is a great exposure to risk factors imposed by this profession, which can significantly affect the work performance and the quality of health of truck drivers. Therefore, changes in their lifestyle are needed and the authorities should draw attention to these professionals.

Descriptors: Worker's Health; Lifestyle; Health Promotion.

\section{RESUMEN}

Objetivo: Analizar la relación del proceso de trabajo y la salud de camioneros del estado de Ceará. Métodos: Se trató de un estudio epidemiológico y transversal desarrollado con 165 camioneros presentes en el XX Festival de los Camioneros del municipio de Tabuleiro del Norte-CE. Para la recogida de datos se aplicó un cuestionario con preguntas cerradas incluyendo los temas relacionados al proceso de trabajo, salud y estilo de vida. Los datos fueron organizados en tablas y analizados en el programa EPI-INFO. Resultados: Se

\author{
Francisco Gilberto Fernandes \\ Pereira $^{(1)}$ \\ Ranúbio Alves de Aquino(2) \\ Valéria Duarte de Melo Alencar ${ }^{(2)}$ \\ Augediva Maria Jucá Pordeus ${ }^{(2)}$ \\ Márcia Barroso Camilo de \\ Ataíde $^{(2)}$
}

1) Centro Universitário Estácio do Ceará Estácio - Fortaleza (CE) - Brasil

2) Universidade de Fortaleza - UNIFOR Fortaleza (CE) - Brasil

Recebido em: 09/07/2014

Revisado em: 19/08/2014

Aceito em: 20/10/2014 
constató que el 100\% $(n=165)$ de los profesionales eran del sexo masculino, el 39,3\% $(n=65)$ estaban en la franja de edad de 30 y 39 años, el 40\% (n=66) tenían solamente la educación primaria incompleta, el 35,8\% (n=59) trabajaban entre12 y 16 horas al dia. Los problemas de salud más comunes fueron el insomnio, en el 78,1\% ( $n=129)$ de los camioneros, con relación significativa con el consumo de sustancias psicoactivas $(p=0,002) ; y$ dolores en la lumbar en el 52,7\% $(n=87)$ de ellos. Conclusión: Hay una gran exposición a los factores de riesgo de parte de esa profesión que puede afectar de modo significativo la ejecución del trabajo y la calidad de la salud de los camioneros. Por lo tanto, se hace necesario cambios en su estilo de vida y más atención a estos profesionales de parte de las autoridades.

Descriptores: Salud Laboral; Estilo de Vida; Salud del Viajero.

\section{INTRODUÇÃO}

As discussões acerca da influência do processo de trabalho e estilo de vida na saúde das pessoas têm ratificado um forte entrelaçamento entre essas variáveis, esclarecendo que muitas das alterações de saúde pelas quais os indivíduos passam estão associadas à forma como reagem aos eventos do trabalho, e o mesmo ocorre com os caminhoneiros ${ }^{(1)}$.

Conforme estudo de avaliação do processo de trabalho e estilo de vida aplicado aos caminhoneiros de estrada, detectou-se que eles são expostos a atividades extenuantes, com longas jornadas diárias de trabalho e condições inadequadas de promoção ou manutenção de saúde, das quais resultam: má alimentação, inatividade física, má quantidade e qualidade de sono, uso indiscriminado de substâncias psicoativas e a solidão(2).

Associam-se ao ritmo intenso de trabalho as longas distâncias a serem percorridas, o cumprimento de horários inflexíveis, as responsabilidades de entrega e seguridade tanto da carga quanto do veículo, e a exposição à insegurança nas estradas. Por esses fatores, os caminhoneiros necessitam dormir na boleia, com alimentação, conforto e segurança precários, acabando por desenvolver um desgaste físicomental, bem como emocional-afetivo, causando variados distúrbios que afetam direta a negativamente sua qualidade de vida $(\mathrm{QV})^{(3)}$.

Na profissão de motorista, não existe rotina diária de trabalho, não há horário previsto para refeição e descanso, ou datas previstas para estar com a família. Em geral, os motoristas não presenciam o nascimento nem acompanham o crescimento dos filhos, convivem com a solidão e a distância da família, buscando o contato social como forma de suavizar o isolamento familiar e de se sentir parte da sociedade $^{(4)}$.
A sobrecarga de trabalho é comum e preocupante nessa atividade laboral, tendo em vista sua influência direta na capacidade de execução dos serviços. E quando não são respeitos os limites do corpo, as consequências podem ser trágicas. Segundo pesquisa feita no ano de 2008 pelo Serviço a Frotas, Eventos e Propagandas da Goodyear do Brasil, 38,2 \% nunca fizeram check-up médico, sendo a falta de tempo o principal motivo alegado. Porém, sabe-se também da dificuldade de se locomover com um caminhão dentro das cidades para procurar assistência, portanto, os caminhoneiros só procuram assistência em casos de emergência ${ }^{(3)}$.

Diante desse contexto de vulnerabilidades, surge a importância de aprofundar a discussão sobre a saúde dos caminhoneiros e conhecer os aspectos peculiares dessa categoria, tendo em vista que esses profissionais são acometidos constantemente por fatores que colocam em risco a sua vida. As ações dirigidas para esse público demandam maior atenção por parte do setor saúde.

Assim, estratégias de promoção da saúde para esse grupo podem surgir a parte deste estudo, contemplando a democratização dos espaços de ofertas dos serviços básicos de saúde e descentralizando-os, de modo a fortalecer, por meio de intervenções e sensibilização educativa: a prevenção de acidentes com múltiplas vítimas, a redução do adoecimento por doenças crônicas não transmissíveis, a proteção contra infecções sexualmente transmissíveis e outras doenças infecciosas que podem ser prevenidas por vacinas.

Portanto, objetivou-se analisar a relação entre processo de trabalho e saúde de caminhoneiros do estado do Ceará.

\section{MÉTODOS}

Estudo epidemiológico transversal desenvolvido com motoristas de caminhão presentes no XX Festival dos Caminhoneiros do município de Tabuleiro do Norte-CE entre os meses de agosto a setembro de 2012.

O município de Tabuleiro do Norte, localizado no Vale do Jaguaribe, a $211 \mathrm{~km}$ de Fortaleza, no estado do Ceará, realiza anualmente, através de sua Associação dos Caminhoneiros (ACATAN), um festival do qual participam cerca de 750 profissionais, que na ocasião foram convidados a participar do estudo no momento em que visitavam o "stand da saúde", instalado no evento pela prefeitura do município.

O cálculo amostral foi realizado utilizando-se amostragem aleatória simples, baseado na população de 750 caminhoneiros previstos para participar do festival. Adotou-se a prevalência de $16,5 \%$ problemas de postura. Considerou-se um erro de $5 \%$ e nível de significância 
de $95 \%$ e $5 \%$ de intervalo de confiança. Após o cálculo amostral, chegou-se ao numero de 165 pessoas.

Incluíram-se no estudo caminhoneiros do sexo masculino, independentemente da idade, pertencentes ao estado do Ceará e que desejaram participar da pesquisa.

Os dados foram obtidos a partir do contato direto estabelecido pelos pesquisadores com os caminhoneiros durante o festival, de forma aleatória, por conveniência, sendo-lhes apresentados os objetivos e relevância do trabalho, formalizando-se o convite para participarem da pesquisa.

Para a coleta de dados, foi aplicado um questionário, elaborado pelos autores e respondido pelos caminhoneiros em uma tenda de atendimento multiprofissional após a abordagem inicial, que constava de perguntas fechadas sobre temas relacionadas ao processo de trabalho, saúde e estilo de vida dos caminhoneiros. No estudo, foram adotadas as variáveis independentes: idade, tempo de trabalho, carga horária, repouso, uso de anfetaminas ou outros medicamentos; e as variáveis dependentes: problemas de saúde como hipertensão, diabetes, insonia, problema de coluna e dependência química.

Os dados foram organizados em tabelas, com o auxílio do programa EPI-INFO. Foi realizada uma análise estatística descritiva com a utilização do mesmo programa.

O desenvolvimento da pesquisa obedeceu aos preceitos éticos da Resolução 466/12 do Ministério da Saúde ${ }^{(5)}$ e todos os participantes da pesquisa assinaram o termo de consentimento livre e esclarecido. A pesquisa foi iniciada após aprovação pelo Comitê de Ética em Pesquisa da Universidade de Fortaleza (UNIFOR), sob o protocolo $\mathrm{n}^{\circ}$ 51832 .

\section{RESULTADOS}

Foram entrevistados 165 caminhoneiros, os quais apresentaram o seguinte perfil sociodemográfico: 100\% $(n=165)$ eram do sexo masculino, $39,3 \%(n=65)$ encontramse na faixa etária de 30 a 39 anos com média de idade de 40 anos, $40 \%(\mathrm{n}=66)$ possuíam apenas o $1^{\circ}$ grau incompleto e $23,6 \%(\mathrm{n}=39)$ declararam ter concluído o $2^{\circ}$ grau.

Com relação ao estado civil, $63,6 \% \quad(n=105)$ se declararam casados e $82,4 \%(n=136)$ declararam ter filhos. Quanto ao convívio com a família durante o período de trabalho, 73,3\% ( $\mathrm{n}=121)$ relataram que algumas vezes viajam com a família e $26,7 \%(n=44)$ nunca trafegam com familiares.

Sobre a renda mensal, 80,6\% $(\mathrm{n}=133)$ declararam possuir renda mensal de 1 até 3 salários mínimos, e apenas $19,3 \%(\mathrm{n}=32)$ referiram ganhar de 4 até 8 salários mínimos mensais. Quanto à propriedade do caminhão, $46,0 \%(\mathrm{n}=76)$ possuem caminhão próprio e $54,0 \%(\mathrm{n}=89)$ afirmam ser o caminhão da empresa para a qual trabalham.

Dos $48,5 \%(n=80)$ de caminhoneiros que referem trabalhar de 12 a 16 horas por dia, $12,7 \%(\mathrm{n}=21)$ declararam ter problemas de insônia e 35,8\% (n=59) disseram que não. Dos caminhoneiros que relatam passar de 11 a 15 dias fora

Tabela I - Distribuição dos caminhoneiros pesquisados segundo relação entre problemas com insônia com horas trabalhadas, horas de sono por dia e qualidade do sono (n=165). Ceará, 2012.

\begin{tabular}{lcccccc}
\hline \multirow{2}{*}{ Variáveis } & \multicolumn{5}{c}{ Problema com insônia } \\
& \multicolumn{2}{c}{ Sim } & \multicolumn{3}{c}{ Não } & \multicolumn{2}{c}{ valores } \\
\cline { 2 - 7 } & $\mathbf{n}$ & $\mathbf{\%}$ & $\mathbf{n}$ & $\mathbf{\%}$ & $\mathbf{x}^{\mathbf{2}}$ & $\boldsymbol{p}$ \\
\hline Horas trabalhadas & & & & & & \\
De 2 a 6 horas & 0 & 0 & 1 & 0,6 & 3,49 & 0,32 \\
De 7 a 11 horas & 5 & 3,1 & 34 & 20,7 & & \\
De 12 a 16 horas & 21 & 12,7 & 59 & 35,8 & & \\
Mais de 16 horas & 13 & 7,8 & 32 & 19,3 & & \\
Horas de sono por dia & & & & & & \\
Até 4 horas & 8 & 4,9 & 29 & 17,6 & 4,67 & 0,19 \\
De 5 a 7 horas & 48 & 29,0 & 48 & 29,0 & & \\
De 8 a 10 horas & 10 & 6,0 & 18 & 11,0 & & \\
De 10 a 12 horas & 0 & 0,0 & 4 & 2,5 & & \\
Qualidade do sono & & & & & & \\
Ótima & 1 & 0,6 & 25 & 15,2 & 31,7 & 0,00 \\
Boa & 5 & 3,0 & 59 & 35,7 & & \\
Regular & 24 & 14,5 & 39 & 23,6 & & \\
Ruim & 4 & 2,5 & 4 & 2,5 & & \\
Péssima & 3 & 1,8 & 1 & 0,6 & & \\
\hline
\end{tabular}

Fonte: Caminhoneiros participantes do XX Festival em Tabuleiro do Norte-CE. Correção de Yates. $x^{2}=$ qui-quadrado 
de casa, 9,6\% $(n=16)$ afirmaram ter problemas de insônia e $35,2 \%(n=58)$ responderam não.

Dos entrevistados que afirmam descansar de 1 a 3 horas durante o dia, 16,9\% $(\mathrm{n}=28)$ referem não ter problemas com insônia e 2,5\% $(n=4)$ afirmaram possuir esse mal. Quando questionados sobre a qualidade do sono, dos entrevistados que consideraram como regular, 14,5\% $(n=24)$ têm problema de insônia e 23,6\% $(n=39)$ dizem não ter esse distúrbio.

Os motoristas de caminhão que costumam consumir algo para não dormir - 34,5\% $(\mathrm{n}=57)$ - responderam que têm problemas de insônia e 7,8\% $(n=13)$ responderam não. Já dos que declararam não consumir nenhum produto para não dormir, $31(18,7 \%)$ citaram ter problemas de insônia e $39,0 \%(n=64)$ não têm problemas (Tabela I).

Houve relação significativa entre o total de 78,1\% $(n=129)$ de caminhoneiros que relataram problemas de insônia com o consumo de substâncias psicoativas $(\mathrm{p}=0,002)$ (Tabela II).

Com relação aos entrevistados que procuraram assistência médica há menos de um ano, 42,3\% $(\mathrm{n}=70)$ não têm problema de insônia e 14,0\% $(n=23)$ têm problema de insônia. A análise da significância estatística entre as variáveis horas trabalhadas, horas de sono por dia e qualidade do sono mostrou que apenas para a qualidade do sono houve relação significativa $(\mathrm{p}=0,00)$ (Tabela I).
Dos 42,2\% $(n=70)$ de caminhoneiros que disseram consumir algo para não dormir, 49,0\% $(n=34)$ consomem medicamentos como a anfetamina, 24,0\% $(n=17)$ usam drogas e $27,0 \%(n=19)$ consomem produtos naturais.

Quanto aos problemas de saúde adquiridos, 83,4\% $(n=64)$ dos participantes relataram enfermidades endócrinometabólicas, como: hipercolesterolemia, hipertensão arterial e diabetes mellitus. Já 59,9\% $(n=99)$ afirmaram ter problemas osteoarticulares, sendo a lombalgia a e artralgia as mais frequentes; $38,1 \%(n=63)$ referiram sofrer de estresse e $78,1 \%(n=129)$ declararam ter problemas de insônia. No que concerne às informaçoes acerca de doenças sexualmente transmissíveis (DSTs), 38,1\% $(n=63)$ declararam que já contraíram-nas, e entre as doenças adquiridas (Tabela II), $67,0 \%(n=42)$ disseram já ter contraído gonorreia, 20,0\% $(n=13)$ herpes, 7,0\% $(n=5)$ sífilis e $6,0 \%(n=3)$ hepatite $B$ e/ou C.

A Tabela III mostra os dados relativos ao trabalho. Observou-se que 29\% $(n=48)$ têm de 11 a 20 anos de profissão, 85,4\% $(\mathrm{n}=141)$ responderam que estão satisfeitos com a profissão, 72,1\% $(n=119)$ declararam que cumprem os prazos estabelecidos na sua profissão, 56,9\% $(n=94)$ já se envolveram em acidente de trânsito e 42,4\% $(n=70)$ foram vítimas de assalto durante o exercício de sua profissão (Tabela III).

Tabela II - Distribuição dos caminhoneiros pesquisados segundo problemas de saúde adquiridos (n=165). Ceará, 2012.

\begin{tabular}{lcc}
\hline Problemas de Saúde* & $\mathbf{n}$ & $\mathbf{\%}$ \\
\hline Alteração de colesterol & 7 & 4,2 \\
Diabetes mellitus & 9 & 5,4 \\
Hipertensão arterial & 48 & 29,0 \\
Problema de articulação & 12 & 7,2 \\
Dores de coluna & 87 & 52,7 \\
Problema de visão & 14 & 8,4 \\
Estresse & 63 & 38,1 \\
Sobrepeso & 74 & 44,8 \\
DST & 63 & 38,1 \\
Insônia** & 129 & 78,1 \\
\hline
\end{tabular}

* Considerar que alguns entrevistados referiram mais de um problema de saúde.

Fonte: Caminhoneiros participantes do XX Festival em Tabuleiro do Norte-CE.

** $\mathrm{p}=0,002$ quando relacionado a utilização de substância psicoativas. 
Tabela III - Distribuição dos caminhoneiros pesquisados segundo eventos relativos à profissão. Ceará, 2012.

\begin{tabular}{lcc}
\hline Variáveis (n=165) & n & \% \\
\hline Anos de profissão & & 18,8 \\
$<5$ anos & 31 & 24,9 \\
5 a 10 anos & 41 & 29,0 \\
11 a 20 anos & 48 & 27,3 \\
Mais de 20 anos & 45 & \\
Gosta da profissão & & 85,4 \\
$\quad$ Sim & 141 & 14,6 \\
$\quad$ Não & 24 & 72,1 \\
Cumprimento dos prazos & 119 & 21,2 \\
$\quad$ Sim & 35 & 6,7 \\
As vezes & 11 & 56,9 \\
Não & & 43,1 \\
Já se envolveu em acidente de trânsito & 94 & 42,4 \\
Sim & 71 & 57,6 \\
$\quad$ Não & & \\
Já foi vítima de assalto & 70 & \\
Sim & 95 & \\
Não & & \\
\hline
\end{tabular}

Fonte: Caminhoneiros participantes do XX Festival em Tabuleiro do Norte-CE.

\section{DISCUSSÃO}

Apesar de existirem algumas mulheres desempenhando a profissão de caminhoneiro, percebeu-se, durante a pesquisa, que ainda é uma atividade executada majoritariamente pela população masculina, com idade predominante entre 30 e 59 anos, resultado semelhante ao identificado em outro estudo com caminhoneiros em região de fronteiras no estado de Rondônia ${ }^{(6)}$.

Muitos dos caminhoneiros entrevistados iniciaram a profissão sem ao menos terem concluído o $1^{\circ}$ grau. Esse é um dado preocupante, tendo em vista que dificilmente esses profissionais retomarão os seus estudos, além de aumentar sua vulnerabilidade aos fatores de risco inerentes à profissão, como acidentes automobilísticos, DSTs, alto consumo de bebidas alcoólicas e uso de drogas ${ }^{(1)}$.

De maneira geral, este estudo revelou que a maioria dos entrevistados $(80,6 \%)$ possuía renda mensal entre 1 e 3 salários mínimos. Dados semelhantes foram apresentados em pesquisa, a qual mostrou que $70 \%$ dos participantes do estudo recebiam de um a cinco salários mínimos ${ }^{(7)}$.

Considerando a relação entre horas trabalhadas e insônia, observamos que, embora a análise dos dados tenha demonstrado significância estatística apenas na qualidade do sono, estudos comprovam que as horas trabalhadas, os dias fora de casa e o consumo de substâncias para não dormir interferem decisivamente na qualidade do sono ${ }^{(8,9)}$, que é de fundamental importância para todo e qualquer ser humano - e na profissão de caminhoneiro não é diferente.
Um descanso adequado é extremamente necessário para que esse profissional desempenhe sua função da melhor maneira possível, de forma que qualquer tipo de comprometimento pode causar graves acidentes em nossas estradas ${ }^{(7)}$.

O estudo mostra que $129(78,1 \%)$ caminhoneiros têm problemas com insônia e $68(41,2 \%)$ consomem algo para não dormir durante a viagem. Esses dados reiteram os resultados apresentados em pesquisa ${ }^{(3)}$ segundo a qual a maioria dos caminhoneiros costuma permanecer, em média, de 12 a 14 horas seguidas ao volante - fator que leva ao consumo de medicamentos estimulantes e dos conhecidos "rebites", muito comuns entre a categoria e facilmente encontrados nos postos e lanchonetes de beira de estrada, os quais servem para manter a pessoa acordada.

A alimentação também é um dos fatores preponderantes, com influência direta na saúde dos caminhoneiros. No entanto, na maioria dos casos, ela é consumida de forma inadequada, acarretando problemas sérios de saúde em médio e longo prazo. Isso já é percebido em um percentual significativo dessa população, pois muitos, ao longo de sua vida, desenvolvem doenças crônicas, como diabetes e hipertensão, afetando sua $\mathrm{QV}^{(4)}$.

Nesse cenário, ações de promoção da saúde indicam um caminho para a melhoria das condições de saúde e de vida da população, apoiando-se no exercício da cidadania. São necessárias, portanto, ações generalizadas que provoquem transformações nos indivíduos, para que passem a atuar como sujeitos dentro da sociedade, tendo a capacidade de atuar no controle das situações às quais são submetidos, isto é, para que atuem como cidadãos ${ }^{(10)}$. 
Dentre os problemas de saúde, o sobrepeso e a hipertensão atingiram um percentual significativo, com $44,8 \%$ e $29,0 \%$, respectivamente. Uma pesquisa conduzida com profissionais de transporte ${ }^{(1)}$ alerta que, em geral, esses profissionais se alimentam em restaurantes à beira de estrada, expostos à maior oferta de alimentos de alto valor calórico e baixo valor nutritivo. Soma-se a isso a alta exposição a bebidas alcoólicas e o sedentarismo, que podem acelerar a ocorrência de doenças como infarto agudo do miocárdio e acidente vascular encefálico.

Os resultados ainda mostram dados preocupantes em relação às doenças adquiridas, chamando atenção para um considerável número de entrevistados (52,7\%) que relataram problemas de coluna. Um estudo ${ }^{(12)}$ reforça que a inadequação do assento é a principal causa das lombalgias. Os tipos de câmbio e direção nos veículos são também elementos ergonômicos importantes para evitar a fadiga do profissional e o surgimento de doenças decorrentes do esforço repetitivo.

No que concerne à ergonomia física, foi criada a Norma Regulamentadora 17 (NR 17), do Ministério do Trabalho e Emprego ${ }^{(13)}$, visando estabelecer parâmetros que permitam a adaptação das condições de trabalho às características psicofisiológicas dos trabalhadores, de modo a proporcionar um máximo de conforto, segurança e desempenho eficiente. Embora tenha sido estabelecida essa NR, é notória a não adequação dessa norma para os caminhoneiros, talvez por desconhecimento de que ela exista ou pela falta de fiscalização. Com isso, surge a necessidade de medidas para punir não só os empregados, mas também os empregadores, tendo em vista que grande parte desses profissionais trabalha em veículos pertencentes a empresas.

A maior parte dos entrevistados refere sofrer de estresse, tendo em vista que, nessa atividade laboral, vários fatores cooperam para esse sintoma, como a dura rotina de trabalho, a ausência da família e as incertezas e dificuldades durante o trajeto nas estradas. Assim, os problemas estressores são constantes, comprometendo bem-estar desse profissional ${ }^{(14)}$.

O estresse é, hoje, um dos principais problemas entre os caminhoneiros. Eles passam várias horas ao volante, atentos ao trânsito, com a carga sob sua responsabilidade, prazos de entrega restritos, risco de acidentes, dívidas e longe da família, ou seja, os sujeitos vivem em constante clima de tensão, sem tempo para lazer, para a prática de atividades físicas e com hábitos alimentares inadequados, levando-os ao adoecimento ${ }^{(4)}$.

A manutenção da saúde ou sua promoção não deve ser somente responsabilidade do setor saúde, mas resultado de ações intersetoriais e multidisciplinares apoiadas por políticas públicas saudáveis, isto é, promotoras de
QV. A saúde valorizada como componente central do desenvolvimento humano ${ }^{(15)}$.

Muitos entrevistados neste estudo $(n=94)$ relataram já ter se envolvido em acidentes. Frente a isso, autores ${ }^{(16)}$ afirmaram que, entre os caminhoneiros de estrada, é bastante comum o uso de anfetaminas para reduzir o sono e diminuir o cansaço em percursos de longa distância, o que pode justificar os acidentes nas estradas. A bebida alcoólica também é muito consumida entre os caminhoneiros, além de ser uma das principais causadoras de acidentes e mortes no trânsito. O uso abusivo dessas substâncias é a principal causa de acidentes e mortes no trânsito, tornando-se um sério problema para saúde pública ${ }^{(12)}$.

Frente à definição da Carta de Ottawa, de que promover saúde é fortalecer e ampliar a capacidade de sujeitos e coletividades de identificar, analisar e exercer controle sobre os determinantes sociais da saúde, assegurando a melhoria das condições e da QV, pode-se dizer que este trabalho deve comprometer-se com a defesa de crescentes graus de autonomia da população, de corresponsabilização pelo cuidado consigo, com os outros e com o mundo ${ }^{(17)}$.

No que se refere à segurança no trabalho, o estudo mostrou que $70(42,4 \%)$ caminhoneiros relataram ter sido vítimas de assalto nos últimos dois anos. Tal fato evidencia a falta de segurança que esses trabalhadores enfrentam, tanto durante o percurso da viagem como nas paradas para o descanso, pois necessitam dormir na boleia do caminhão, à beira de estradas ou em postos de gasolina, os quais não oferecem nenhum tipo de segurança, sendo muitas vezes surpreendidos por assaltantes.

Uma pesquisa realizada no Brasil com 513 caminhoneiros $^{(18)}$ evidenciou duas grandes queixas dos caminhoneiros: a segurança e o papel da polícia. Muitos afirmaram que o policiamento é ineficiente, pois não previne os assaltos; além disso, afirmaram que os policiais são corruptos, ao extorquirem dinheiro por multas abusivas.

Neste estudo, foi detectado que $63 \quad(38,1 \%)$ caminhoneiros já contraíram algum tipo de DST, sendo a gonorreia, herpes, sífilis e hepatite $\mathrm{B}$ e/ou $\mathrm{C}$ as mais citadas. A vulnerabilidade do caminhoneiro às DSTs/AIDS não está somente associada ao tempo que ele permanece fora de casa, mas à cultura inerente a essa categoria. Um estudo cita alguns aspectos culturais, próprios do gênero masculino, que tornam os homens mais vulneráveis às práticas de risco para as DSTs/AIDS, tais como: sentir-se forte, imune a doenças; ser impetuoso, correr riscos; ser incapaz de recusar uma mulher; considerar que o homem tem mais necessidade de sexo do que a mulher e de que esse desejo é incontrolável. A infidelidade masculina é considerada natural; a feminina é atribuída a deficiências do parceiro ${ }^{(4)}$. 
A maioria dos entrevistados deste estudo $(85,4 \%)$ respondeu que são felizes com o trabalho, dados semelhantes aos de outra pesquisa ${ }^{(7)}$, na qual $85 \%$ dos participantes responderam gostar da profissão de caminhoneiro. Como aspectos positivos da profissão, boa parte dos caminhoneiros destacou as manifestações sobre o estilo comportamental de vida, em busca de novas experiências, conhecer lugares, pessoas diferentes e até mesmo ter contato com outras mulheres. Entre aqueles que relataram gostar da profissão, estão pessoas realmente apaixonadas pelo que fazem, por ser uma profissão em que se ganha bem e por ter herdado a profissão do pai ${ }^{(6)}$.

Diante dos resultados encontrados na presente pesquisa, constatou-se a necessidade da criação de ações que possam trazer melhorias na $\mathrm{QV}$ de caminhoneiros e da implantação de políticas de saúde que possam garantir a esse público o direito de serem assistidos na sua integralidade, proporcionando melhores condições de trabalho e maior cobertura assistencial. No entanto, para que isso ocorra, faz-se necessário que as autoridades de saúde encarem esse desafio com seriedade, certificando-se para que medidas efetivas sejam adotadas com o propósito de melhorar a qualidade e o estilo de vida dessa população, para que possam ter condições dignas de trabalho. Portanto, compreende-se a promoção da saúde como uma possibilidade de ampliação das oportunidades de produzir a saúde dos caminhoneiros.

\section{CONCLUSÃO}

Existe uma grande exposição a fatores de risco imposta por essa profissão, podendo afetar de maneira significativa a execução do trabalho e a qualidade da saúde dos caminhoneiros. Portanto, tornam-se necessárias mudanças no estilo de vida destes e que as autoridades dirijam maior atenção para esses profissionais.

\section{REFERÊNCIAS}

1. Takiane J, Oliveira LG, Endo LG, Oliveira KCBG, Munoz DR, Yonamime M, et al. Amphetamine use by truck drivers on highways of São Paulo State: a risk for the occurrence of traffic acidentes? Ciênc Saúde Coletiva. 2013;18(5):1247-54.

2. Knauth DR, Pilecco FB, Leal AF, Seffner F, Teixeira AMFB. Manter-se acordado: a vulnerabilidade dos caminhoneiros no Rio Grande do Sul. Rev Saúde Pública 2012;46(5):886-93.

3. Sedano GS, Ferreira SCM, Valente GSC, Chrisostimo MM. Educação em saúde: um desafio do enfermeiro do trabalho na atenção à saúde dos caminhoneiros. Rev Pesqui Cuid Fundam (Impr.). 2010;2(2):760-9.
4. Masson VA, Monteiro MI. Estilo de vida, aspectos de saúde e trabalho de motoristas de caminhão. Rev Bras Enferm. 2010;63(4):533-40.

5. Ministério da Saúde (BR). Resolução $n^{0}$ 466/12. Brasília: Ministério da Saúde; 2012.

6. Rocha EM. DST e Aids em região de fronteira: um estudo com caminhoneiros no estado de Rôndonia [dissertação]. Brasília: Universidade de Brasília; 2007.

7. Resende PTV, Sousa PR, Cerqueira PR. Hábitos de vida e segurança dos caminhoneiros brasileiros [resumo]. In: Simpósio de Administração da Produção, Logística e Operações Internacionais. Anais do SIMPOI 2010; 2010 Ago. São Paulo: FGV; 2010. p. 1-17

8. Xavier KGS, Vaghetti HH. Aspectos cronobiológicos do sono de enfermeiras de um hospital universitário. Rev Bras Enferm. 2012;65(1):135-40.

9. Zarpelao RZN, Martino MMF. A qualidade do sono e os trabalhadores de turno: revisão integrativa. Rev Enferm UFPE. 2014;8(6):1782-90.

10. Haeser LM, Buchele F, Brzozowski FS. Considerações sobre a autonomia e a promoção da saúde. Physis. 2012;22(2):605-20.

11. Cavagioni LC. Perfil dos riscos cardiovasculares em motoristas profissionais de transportes de carga da rodovia BR-116 no trecho paulista-Régis Bittencourt [dissertação]. São Paulo: Escola de Enfermagem Universidade de São Paulo; 2006.

12. Salles GCS, Pereira CA, Passos JP. Condições de trabalho dos profissionais de transporte e sua relação com a saúde. Rev Pesqui Cuid Fundam (Impr.). 2011;3(1):1692-700.

13. Ministério do Trabalho e Emprego (BR). Norma Regulamentadora $\mathrm{n}^{\circ}$ 17- Ergonomia. Brasília: Ministério do Trabalho e Emprego; 2007.

14. Alquimim AF, Barral ABCR, Gomes KC, Rezende MC. Avaliação dos fatores de risco laborais e físicos para doenças cardiovasculares em motoristas de transporte urbano de ônibus em Montes Claros (MG). Ciênc Saúde Coletiva. 2012;17(8):2151-8.

15. Iglesias A, Dabello-Araujo M. As concepções de promoção da saúde e suas implicações. Cad Saúde Coletiva. 2011;19(3):291-8.

16. Nascimento EC, Nascimento E, Silva JP. Uso de álcool e anfetaminas entre caminhoneiros de estrada. Rev Saúde Pública. 2006;41(2):290-3.

17. Campos GWS, Barros RB, Castro AM. Avaliação de política nacional de promoção da saúde. Ciênc Saúde Coletiva. 2004;9(3):745-9. 
18. Koller AS. Vida dos caminhoneiros brasileiros. Projeto de pesquisa: A vida dos caminhoneiros brasileiros. Sweden: WCF Foundation Brazil; 2005.

\section{Endereço para correspondência:}

Francisco Gilberto Fernandes Pereira

Rua: Prof. Vicente Silveira, 100, Bl 2, Ap 404

Bairro: Vila União

CEP: 60410-322 - Fortaleza - CE - Brasil

E-mail: gilberto.fp@hotmail.com 